\title{
Al for Pulmonary Abnormality Screening: TB, COVID-19, and Many More
}

\author{
Dr. KC Santosh \\ University of South Dakota, USA.
}

With the advent of new technologies in machine learning and AI, computer scientists often do not respect the data sentiments as they are trying to lowering down the learning error-rate. Conventionally speaking, from machine learning perspective, lowering down the learning error-rates yields higher recognition rate. However, if we consider expert-based problems, such as healthcare, it is wise to follow and/or replicate expert-based procedure; but procedural solution is out of fashion. In this talk, we will be having multiple projects on lung abnormality screening using image data (chest X-ray). Considering two different cases, TB and COVID-19, we will be discussing on a) do medical imaging scientists care clinical implications?; b) is it required to replicate the experts' procedures to make decisions? If not, can we be that much ignorant?; c) how big data is big for computer scientists?; and d) when can we deploy imaging tools for cross-population tests? 